\title{
Singular optical solitons in birefringent nano-fibers
}

Michelle Savescu ${ }^{1}$, Qin Zhou ${ }^{2, *}$, Luminita Moraru ${ }^{3}$, Anjan Biswas ${ }^{4,5,6}$, Seithuti P. Moshokoa ${ }^{6}$, Milivoj Belic ${ }^{7}$

${ }^{1}$ Department of Mathematics, Kuztown University of Pennsylvania, 15200 Kutztown Road, Kuztown, PA-19530, USA

${ }^{2}$ School of Electronics and Information Engineering, Wuhan Donghu University, Wuhan, 430212, P.R. China

${ }^{3}$ Faculty of Sciences and Environment, Department of Chemistry, Physics and Environment, Dunarea de Jos University of Galati, 47 Domneasca St., 800008, Romania

${ }^{4}$ PO Box 137; Cheswold, DE-19936; USA

${ }^{5}$ Department of Mathematics, Faculty of Science, King Abdulaziz University, Jeddah-21589, Saudi Arabia

${ }^{6}$ Department of Mathematics and Statistics, Tshwane University of Technology, Pretoria0008, South Africa

${ }^{7}$ Science Program, Texas A and M University at Qatar, PO Box 23874, Doha, Qatar

\begin{abstract}
This paper serves as a sequel to previously published results on bright, dark and singular solitons in birefringent fibers with spatio-temporal dispersion, during 2014. The second form of singular soliton solutions is retrieved in this paper for birefringent nano-fibers with Kerr and parabolic laws of nonlinearity. There are constraint conditions that evolve with the solution structure.
\end{abstract}

Key words: birefringence; integrability; singular solitons. 


\section{INTRODUCTION}

Optical solitons is one of the fastest growing areas of research in the field of nonlinear optics and telecommunications engineering. There are several overwhelming results that are constantly pouring into several journals from all across the globe [1-32]. These results are gradually leading to advances and successful achievements in telecommunications. The modern day engineering marvels such as Internet industry, face-book, and twitter are possible only with soliton communication technology.

Therefore this area of research, namely optical solitons is being further probed into, constantly, from all avenues and all angles. One aspect of soliton propagation through nonlinear optical fibers is that results from vector solitons are less visible. This includes solitons from birefringent fibers, cascaded system, thirring solitons, DWDM systems and others. This paper is going to address solitons in birefringent fibers. Very recently, bright, dark and singular soliton solutions in birefringent fibers with Kerr law and parabolic law nonlinearity, in presence of Hamiltonian perturbation terms, are reported [1]. This paper is going to study the same model with a second form of singular soliton solution for both of these nonlinear media. There are several constraint conditions that will be listed for these solitons to exist.

For this model, as reported in 2014 [1], the governing nonlinear Schrödinger's equation will be considered with spatio-temporal dispersion (STD) in addition to group velocity dispersion (GVD). The inclusion of STD only makes the model well-posed as pointed out during $2012[5,8]$. Another aspect for considering STD, in addition to GVD, is the reduction of Internet bottleneck.

The mathematical analysis of singular soliton solutions will be carried out in the rest of the paper. The study will be split into subsequent two sections with Kerr law and parabolic law nonlinearity. 


\section{KERR LAW}

This section addresses the bright, dark as well as singular solitons in optical fibers with Kerr law nonlinearity. The dimensionless form of the coupled NLSE with STD and Kerr law nonlinearity is given by $[1,3]$

$i q_{t}+a_{1} q_{x x}+b_{1} q_{x t}+\left(c_{1}|q|^{2}+d_{1}|r|^{2}\right) q+i\left\{\alpha_{1} q_{x}+\lambda_{1}\left(|q|^{2} q\right)_{x}+\right.$

$\left.v_{1}\left(|q|^{2}\right)_{x} q+\theta_{1}|q|^{2} q_{x}+\gamma_{1} q_{x x x}\right\}=0$

$i r_{t}+a_{2} r_{x x}+b_{2} r_{x t}+\left(c_{2}|r|^{2}+d_{1}|q|^{2}\right) r+i\left\{\alpha_{2} r_{x}+\lambda_{2}\left(|r|^{2} r\right)_{x}+\right.$

$\left.v_{2}\left(|r|^{2}\right)_{x} r+\theta_{2}|r|^{2} r_{x}+\gamma_{2} r_{x x x}\right\}=0$

In (1) and (2) $q(x, t)$ and $r(x, t)$ are complex valued functions that represents the soliton profiles for the two components in birefringent fibers. For $l=1 ; 2, a_{l}$ represents the GVD and $b_{l}$ are STD terms along the two components. Then, $c_{l}$ and $d_{l}$ represents the self-phase modulation (SPM) and cross-phase modulation (XPM) terms respectively. In the perturbation terms $\alpha_{l}$ represents the inter-modal dispersion, $\lambda_{l}$ is the self-steepening term, $v_{l}$ and $\theta_{l}$ are nonlinear dispersions and finally $\gamma_{l}$ is the third order dispersion that must be taken into account in case the GVD is small $[1,3,7]$.

The aim of this section is to obtain an exact bright, dark and singular 1-soliton solution to this coupled set of equations. The ansatz method is used. In order to set up the starting point, the solitons are written in the phase-amplitude format as $[1,16,17]$

$$
q(x, t)=A_{1} \operatorname{coth}^{p_{1}}[B(x-v t)] e^{i\left(-\kappa_{1}+\omega_{1} t+\sigma_{1}\right)}=A_{1} \operatorname{coth}^{p_{1}} \tau e^{i \Phi_{1}(x, t)}
$$

and

$$
r(x, t)=A_{2} \operatorname{coth}^{p_{2}}[B(x-v t)] e^{i\left(-\kappa_{2}+\omega_{2} t+\sigma_{2}\right)}=A_{2} \operatorname{coth}^{p_{2}} \tau e^{i \Phi_{2}(x, t)}
$$

where $A_{l}$ and $B$ are free parameters, while $\Phi_{l}(x, t)$ are phase components, defined as

$$
\Phi_{l}(x, t)=-\kappa_{l}+\omega_{l} t+\sigma_{l}
$$

For $l=1 ; 2$. Also, $\tau$ is given by

$$
\tau=B(x-v t)
$$


Here, $\kappa_{l}$ represents frequency of solitons for each of the two components, $\omega_{l}$ are the wave numbers, while $\sigma_{l}$ are the phase constants. Substituting (3) and (4) into (1) and (2) and decomposing into real and imaginary parts lead to

$$
\begin{aligned}
& \left\{\omega_{l}\left(b_{l} \kappa_{l}-1\right)-a_{l} \kappa_{l}^{2}+\alpha_{l} \kappa_{l}-\gamma_{l} \kappa_{l}^{3}-4 p_{l}^{2}\left(a_{l}+3 \gamma_{l} \kappa_{l}\right) B^{2}+2 p_{l}\left(p_{l}-2\right) v b_{l} B^{2}\right\} \operatorname{coth}^{2} \tau+ \\
& d_{l} A_{\bar{l}}^{2} \operatorname{coth}^{2 p_{\bar{l}}+2} \tau+\left(\lambda_{l} \kappa_{l}+\theta_{l} \kappa_{l}+c_{l}\right) A_{l}^{2} \operatorname{coth}^{2 p_{l}+2} \tau+p_{l}\left\{\left(p_{l}+1\right)\left(a_{l}+3 \gamma_{l} \kappa_{l}\right)-\right. \\
& \left.\left(p_{l}-3\right) v b_{l}\right\} B^{2} \operatorname{coth}^{4} \tau+p_{l}\left(p_{l}-1\right)\left(a_{l}+3 \gamma_{l} \kappa_{l}-v b_{l}\right) B^{2}=0
\end{aligned}
$$

and

$\left\{v\left(1-b_{l} \kappa_{l}\right)-2 a_{l} \kappa_{l}+b_{l} \omega_{l}+\alpha_{l}-\gamma_{l}\left(3 \kappa_{l}^{2}+2 p_{l}^{2} B^{2}\right)\right\} \operatorname{coth}^{2} \tau+\gamma_{l}\left(p_{l}^{2}+3 p_{l}+\right.$

2) $B^{4} \operatorname{coth}^{4} \tau+\left(3 \lambda_{l}+2 v_{l}+\theta_{l}\right) A_{l}^{2} \operatorname{coth}^{2 p_{l}+2} \tau+\gamma_{l}\left(p_{l}-1\right)\left(p_{l}-2\right) B^{2}=0$,

respectively. Application of balancing principle to (7) and (8) reveals

$$
p_{l}=1
$$

for $l=1 ; 2$ and $\bar{l}=3-l$.

From the imaginary part equation (8), upon setting the coefficients of the linearly independent function to zero yields the speed of the soliton as

$$
v=\frac{2 a_{l} \kappa_{l}-b_{l} \omega_{l}-\alpha_{l}+\gamma_{l}\left(3 \kappa_{l}^{2}+2 B^{2}\right)}{b_{l} \kappa_{l}-1},
$$

for

$$
b_{l} \kappa_{l} \neq 1
$$

Now, equating the two components of speed gives the free parameter

$$
B=\left[\frac{\left(b_{1} \kappa_{1}-1\right)\left(2 a_{2} \kappa_{2}-b_{2} \omega_{2}-\alpha_{2}+3 \gamma_{2} \kappa_{2}^{2}\right)-\left(b_{2} \kappa_{2}-1\right)\left(2 a_{1} \kappa_{1}-b_{1} \omega_{1}-\alpha_{1}+3 \gamma_{1} \kappa_{1}^{2}\right)}{2\left\{\gamma_{1}\left(b_{2} \kappa_{2}-1\right)-\gamma_{2}\left(b_{1} \kappa_{1}-1\right)\right\}}\right]^{\frac{1}{2}}
$$

subject to the constraint:

$$
\begin{aligned}
& \gamma_{1}\left(b_{2} \kappa_{2}-1\right)-\gamma_{2}\left(b_{1} \kappa_{1}-1\right) \times \\
& \times\left\{\left(b_{1} \kappa_{1}-1\right)\left(2 a_{2} \kappa_{2}-b_{2} \omega_{2}-\alpha_{2}+3 \gamma_{2} \kappa_{2}^{2}\right)-\left(b_{2} \kappa_{2}-1\right)\left(2 a_{1} \kappa_{1}-b_{1} \omega_{1}-\alpha_{1}+3 \gamma_{1} \kappa_{1}^{2}\right)\right\}>0
\end{aligned}
$$

The coefficient of second linearly independent function gives the free parameter 


$$
A_{l}=\left[\frac{3 \gamma_{l}\left\{\left(b_{l} \kappa_{l}-1\right)\left(2 a_{\bar{l}} \kappa_{\bar{l}}-b_{\bar{l}} \omega_{\bar{l}}-\alpha_{\bar{l}}+3 \gamma_{\bar{l}} \kappa_{\bar{l}}^{2}\right)-\left(b_{\bar{l}} \kappa_{\bar{l}}-1\right)\left(2 a_{l} \kappa_{l}-b_{l} \omega_{l}-\alpha_{l}+3 \gamma_{l} \kappa_{l}^{2}\right)\right\}}{\left(3 \lambda_{l}+2 v_{l}+\theta_{l}\right)\left\{\gamma_{\bar{l}}\left(b_{l} \kappa_{l}-1\right)-\gamma_{l}\left(b_{\bar{l}} \kappa_{\bar{l}}-1\right)\right\}}\right]^{\frac{1}{2}}
$$

provided

$$
\begin{aligned}
& \gamma_{l}\left\{\left(b_{l} \kappa_{l}-1\right)\left(2 a_{\bar{l}} \kappa_{\bar{l}}-b_{\bar{l}} \omega_{\bar{l}}-\alpha_{\bar{l}}+3 \gamma_{\bar{l}} \kappa_{\bar{l}}^{2}\right)-\left(b_{\bar{l}} \kappa_{\bar{l}}-1\right)\left(2 a_{l} \kappa_{l}-b_{l} \omega_{l}-\alpha_{l}+3 \gamma_{l} \kappa_{l}^{2}\right)\right\} \times \\
& {\left[\left(3 \lambda_{l}+2 v_{l}+\theta_{l}\right)\left\{\gamma_{\bar{l}}\left(b_{l} \kappa_{l}-1\right)-\gamma_{l}\left(b_{\bar{l}} \kappa_{\bar{l}}-1\right)\right\}>0\right]}
\end{aligned}
$$

Next, the real part equation (7) gives, in a similar manner,

$$
v=\frac{2\left(a_{l}+3 \gamma_{l} \kappa_{l}\right) B^{2}+\left(\lambda_{l} \kappa_{l}+\theta_{l} \kappa_{l}+c_{l}\right) A_{l}^{2}+d_{l} A_{\bar{l}}^{2}}{2 b_{l} B^{2}}
$$

and the wave number

$$
\omega_{l}=\frac{a_{l} \kappa_{l}^{2}-\alpha_{l} \kappa_{l}+\gamma_{l} \kappa_{l}^{3}-\left(\lambda_{l} \kappa_{l}+\theta_{l} \kappa_{l}+c_{l}\right) A_{l}^{2}-d_{l} A_{l}^{2}}{b_{l} \kappa_{l}-1}
$$

with the constraint given by (11).

Finally, substituting the free parameter $A_{l}$ from (14) into (17) leads to the coupled system of equation for the frequencies of the two components:

$$
\left[\begin{array}{ll}
P^{(1)} & Q^{(1)} \\
P^{(2)} & Q^{(2)}
\end{array}\right]\left[\begin{array}{l}
\omega_{1} \\
\omega_{2}
\end{array}\right]=\left[\begin{array}{l}
R^{(1)} \\
R^{(2)}
\end{array}\right]
$$

where

$$
\begin{aligned}
& P^{(l)}=1-\frac{3 b_{l}\left(b_{\bar{l}} \kappa_{\bar{l}}-1\right)}{\left(b_{l} \kappa_{l}-1\right)\left\{\gamma_{l}\left(b_{\bar{l}} \kappa_{\bar{l}}-1\right)-\gamma_{\bar{l}}\left(b_{l} \kappa_{l}-1\right)\right\}}\left[\frac{d_{l} \gamma_{\bar{l}}}{3 \lambda_{\bar{l}}+2 v_{\bar{l}}+\theta_{\bar{l}}}+\frac{\gamma_{l}\left(\lambda_{l} \kappa_{l}+\theta_{l} \kappa_{l}+c_{l}\right)}{3 \lambda_{l}+2 v_{l}+\theta_{l}}\right] \\
& Q^{(l)}=\frac{3 b_{\bar{l}}}{\left\{\gamma_{l}\left(b_{\bar{l}} \kappa_{\bar{l}}-1\right)-\gamma_{\bar{l}}\left(b_{l} \kappa_{l}-1\right)\right\}}\left[\frac{d_{l} \gamma_{\bar{l}}}{3 \lambda_{\bar{l}}+2 v_{\bar{l}}+\theta_{\bar{l}}}+\frac{\gamma_{l}\left(\lambda_{l} \kappa_{l}+\theta_{l} \kappa_{l}+c_{l}\right)}{3 \lambda_{l}+2 v_{l}+\theta_{l}}\right]
\end{aligned}
$$

and

$$
\begin{aligned}
& R^{(l)}=\frac{a_{l} \kappa_{l}^{2}-\alpha_{l} \kappa_{l}+\gamma_{l} \kappa_{l}^{3}}{b_{l} \kappa_{l}-1}+ \\
& +3\left\{\left(2 a_{\bar{l}} \kappa_{\bar{l}}-\alpha_{\bar{l}}+3 \gamma_{\bar{l}} \kappa_{\bar{l}}^{2}\right)\left(b_{l} \kappa_{l}-1\right)-\left(2 a_{l} \kappa_{l}-\alpha_{l}+3 \gamma_{l} \kappa_{l}^{2}\right)\left(b_{\bar{l}} \kappa_{\bar{l}}-1\right)\right\} \times \\
& \times\left[\frac{d_{l} \gamma_{\bar{l}}}{3 \lambda_{\bar{l}}+2 v_{\bar{l}}+\theta_{\bar{l}}}+\frac{\gamma_{l}\left(\lambda_{l} \kappa_{l}+\theta_{l} \kappa_{l}+c_{l}\right)}{3 \lambda_{l}+2 v_{l}+\theta_{l}}\right]
\end{aligned}
$$


for $l=1 ; 2$ and $\bar{l}=3-l$. Inverting the matrix in (18) leads to the frequencies of the two components:

$$
\left[\begin{array}{c}
\omega_{1} \\
\omega_{2}
\end{array}\right]=\frac{1}{P^{(1)} Q^{(2)}-P^{(2)} Q^{(1)}}\left[\begin{array}{l}
Q^{(2)} R^{(1)}-Q^{(1)} R^{(2)} \\
P^{(1)} R^{(2)}-P^{(2)} R^{(1)}
\end{array}\right]
$$

as long as

$$
\left[\begin{array}{ll}
P^{(1)} & Q^{(1)} \\
P^{(2)} & Q^{(2)}
\end{array}\right] \neq 0
$$

Hence, singular 1-soliton solutions to (1) and (2) are given by

$$
\begin{aligned}
& q(x, t)=A_{1} \operatorname{coth}[B(x-v t)] e^{i\left(-\kappa_{1}+\omega_{1} t+\sigma_{1}\right)} \\
& r(x, t)=A_{2} \operatorname{coth}[B(x-v t)] e^{i\left(-\kappa_{2}+\omega_{2} t+\sigma_{2}\right)}
\end{aligned}
$$

with all parameters as indicated.

\section{PARABOLIC LAW}

For parabolic law nonlinearity, the governing equations in birefringent nano-fibers are given by the following coupled system $[1,3,6]$ :

$$
\begin{aligned}
& i q_{t}+a_{1} q_{x x}+b_{1} q_{x t}+\left(c_{1}|q|^{2}+d_{1}|r|^{2}\right) q+\left(\xi_{1}|q|^{4}+\eta_{1}|q|^{2}|r|^{2}+\right. \\
& \left.\zeta_{1}|q|^{4}\right)+i\left\{\alpha_{1} q_{x}+\lambda_{1}\left(|q|^{2} q\right)_{x}+v_{1}\left(|q|^{2}\right)_{x} q+\theta_{1}|q|^{2} q_{x}+\gamma_{1} q_{x x x}\right\}=0 \\
& i r_{t}+a_{2} r_{x x}+b_{2} r_{x t}+\left(c_{2}|r|^{2}+d_{2}|q|^{2}\right) r+\left(\xi_{2}|r|^{4}+\eta_{2}|r|^{2}|q|^{2}+\right. \\
& \left.\zeta_{2}|q|^{4}\right)+i\left\{\alpha_{2} r_{x}+\lambda_{2}\left(|r|^{2} r\right)_{x}+v_{2}\left(|r|^{2}\right)_{x} r+\theta_{2}|r|^{2} r_{x}+\gamma_{2} r_{x x x}\right\}=0
\end{aligned}
$$

In order to seek singular soliton solutions of second type, for birefringent fibers with parabolic law, the starting hypothesis is taken to be $[16,17,19,20]$ :

$$
q(x, t)=\left\{A_{1}+B_{1} \operatorname{coth}[B(x-v t)]\right\}^{p_{1}} e^{i\left(-\kappa_{1}+\omega_{1} t+\sigma_{1}\right)}=\left(A_{1}+B_{1} \operatorname{coth} \tau\right)^{p_{1}} e^{i \Phi_{1}(x, t)}
$$

and

$$
r(x, t)=\left\{A_{2}+B_{2} \operatorname{coth}[B(x-v t)]\right\}^{p_{2}} e^{i\left(-\kappa_{2}+\omega_{2} t+\sigma_{2}\right)}=\left(A_{2}+B_{2} \operatorname{coth} \tau\right)^{p_{2}} e^{i \Phi_{2}(x, t)}
$$


Once again, $A_{l}$ and $B_{l}$ are free parameters. Substituting (28) and (29) into (26) and (27), and decomposing into real and imaginary parts gives a pair of relations. The imaginary part leads to

$$
\begin{aligned}
& \gamma_{l} B^{2}\left(p_{l}-1\right)\left(p_{l}-2\right)\left(B_{l}^{2}-A_{l}^{2}\right)^{2}+2\left(2 p_{l}^{2}-3 p_{l}+1\right) \gamma_{l} A_{l} B^{2}\left(B_{l}^{2}-A_{l}^{2}\right)\left(A_{l}+B_{l} \operatorname{coth} \tau\right)+ \\
& +\left[B_{l}^{2}\left\{\left(b_{l} \kappa_{l}-1\right) v-2 a_{l} \kappa_{l}+b_{l} \omega_{l}+\alpha_{l}-3 \gamma_{l}^{2}\right\}+2 \gamma_{l} B^{2} p_{1}^{2}\left(3 A_{l}^{2}-B_{l}^{2}\right)\right]\left(A_{l}+B_{l} \operatorname{coth} \tau\right)^{2}- \\
& -2 \gamma_{l} B^{2} A_{l}\left(2 p_{l}^{2}+3 p_{l}+1\right)\left(A_{l}+B_{l} \operatorname{coth} \tau\right)^{3}+\gamma_{l} B^{2}\left(p_{l}^{2}-9 p_{l}+14\right)\left(A_{l}+B_{l} \operatorname{coth} \tau\right)^{4}+ \\
& \left(3 \lambda_{l}+2 v_{l}+\theta_{l}\right)\left(A_{l}+B_{l} \operatorname{coth} \tau\right)^{2 p_{l}+2}=0
\end{aligned}
$$

From the linearly independent functions, it shows that third-order dispersion must vanish, namely

$$
\gamma_{l}=0
$$

and also

$$
A_{l}=B_{l}
$$

Equation (31) shows that singular solitons of second type, in birefringent fibers, with parabolic law nonlinearity, will exist, provided third order dispersion vanishes, which is contrary to Kerr law nonlinearity. This observation is being made for the first time in this paper.

Now, the governing equations (26) and (27), by virtue of (31), reduces to

$$
\begin{aligned}
& i q_{t}+a_{1} q_{x x}+b_{1} q_{x t}+\left(c_{1}|q|^{2}+d_{1}|r|^{2}\right) q+\left(\xi_{1}|q|^{4}+\eta_{1}|q|^{2}|r|^{2}+\right. \\
& \left.\zeta_{1}|q|^{4}\right)+i\left\{\alpha_{1} q_{x}+\lambda_{1}\left(|q|^{2} q\right)_{x}+v_{1}\left(|q|^{2}\right)_{x} q+\theta_{1}|q|^{2} q_{x}\right\}=0 \\
& i r_{t}+a_{2} r_{x x}+b_{2} r_{x t}+\left(c_{2}|r|^{2}+d_{2}|q|^{2}\right) r+\left(\xi_{2}|r|^{4}+\eta_{2}|r|^{2}|q|^{2}+\right. \\
& \left.\zeta_{2}|q|^{4}\right)+i\left\{\alpha_{2} r_{x}+\lambda_{2}\left(|r|^{2} r\right)_{x}+v_{2}\left(|r|^{2}\right)_{x} r+\theta_{2}|r|^{2} r_{x}\right\}=0
\end{aligned}
$$

respectively. The remaining linearly independent functions, from (30), lead to the constraint

$$
3 \lambda_{l}+2 v_{l}+\theta_{l}=0
$$

and the speed of the soliton

$$
v=\frac{2 a_{l} \kappa_{l}-b_{l} \omega_{l}-\alpha_{l}}{b_{l} \kappa_{l}-1}
$$


for $l=1 ; 2$. Now, equating the two expressions for the speed of the soliton from (36), for $l=$ 1; 2 implies the constraint:

$$
\left(2 a_{1} \kappa_{1}-b_{1} \omega_{1}-\alpha_{1}\right)\left(b_{2} \kappa_{2}-1\right)=\left(2 a_{2} \kappa_{2}-b_{2} \omega_{2}-\alpha_{2}\right)\left(b_{1} \kappa_{1}-1\right)
$$

Next, the real part equation, after setting $\gamma_{l}=0$, simplifies to:

$B^{2} p_{l}\left(p_{l}+1\right)\left(a_{l}-b_{l} v\right)\left(A_{l}+B_{l} \operatorname{coth} \tau\right)^{2}-2 B^{2} A_{l} p_{l}\left(2 p_{l}+1\right)\left(a_{l}-b_{l} v\right)\left(A_{l}+B_{l} \operatorname{coth} \tau\right)^{3}+$ $\left[B_{l}^{2}\left\{\omega_{l}\left(b_{l} \kappa_{l}-1\right)-a_{l} \kappa_{l}^{2}+\alpha_{l} \kappa_{l}\right\}+2 B^{2} p_{l}^{2}\left(a_{l}-b_{l} v\right)\left(3 A_{l}^{2}-B_{l}^{2}\right)\right]\left(A_{l}+B_{l} \operatorname{coth} \tau\right)^{2}+$ $2 p_{l}\left(2 p_{l}-1\right) B^{2} A_{l}\left(a_{l}-b_{l} v\right)\left(B_{l}^{2}-A_{l}^{2}\right)\left(A_{l}+B_{l} \operatorname{coth} \tau\right)+B^{2} p_{l}\left(p_{l}-1\right)\left(a_{l}-b_{l} v\right)\left(B_{l}^{2}-\right.$ $\left.A_{l}^{2}\right)^{2}+\left(\lambda_{l} \kappa_{l}+\theta_{l} \kappa_{l}+c_{l}\right)\left(A_{l}+B_{l} \operatorname{coth} \tau\right)^{2 p_{l}+2}+d_{l}\left(A_{l}+B_{l} \operatorname{coth} \tau\right)^{2}\left(A_{\bar{l}}+B_{\bar{l}} \operatorname{coth} \tau\right)^{2 p_{\bar{l}}}+$ $\xi_{l}\left(A_{l}+B_{l} \operatorname{coth} \tau\right)^{4 p_{l}+2}+\eta_{l}\left(A_{l}+B_{l} \operatorname{coth} \tau\right)^{2 p_{l}+2}\left(A_{\bar{l}}+B_{\bar{l}} \operatorname{coth} \tau\right)^{2 p_{\bar{l}}}+$ $\zeta_{l}\left(A_{l}+B_{l} \operatorname{coth} \tau\right)^{2}\left(A_{\bar{l}}+B_{\bar{l}} \operatorname{coth} \tau\right)^{4 p_{\bar{l}}}=0$

Balancing principle indicates

$$
p_{l}=\frac{1}{2}
$$

for $l=1 ; 2$. From (38), setting the coefficients of linearly independent functions to zero yields the wave number and the free parameter B as

$$
\omega_{l}=\frac{\kappa_{l}\left(a_{l} \kappa_{l}-\alpha_{l}\right)+\left(b_{l} v-a_{l}\right) B^{2}}{b_{l} \kappa_{l}-1}
$$

and

$$
B=\frac{\left(\lambda_{l} \kappa_{l}+\theta_{l} \kappa_{l}+c_{l}\right) A_{l}}{2\left(a_{l}-b_{l} v\right)}
$$

respectively, together with the condition

$$
\left(\lambda_{l} \kappa_{l}+\theta_{l} \kappa_{l}+c_{l}\right)\left(a_{l}-b_{l} v\right) A_{l}>0
$$

Another expression for the wave number, that falls out from the linearly independent function is

$$
\omega_{l}=\frac{3 \kappa_{l}\left(a_{l} \kappa_{l}-\alpha_{l}\right)+4 \xi_{l} A_{l}^{2}}{b_{l} \kappa_{l}-1}
$$

The relations (40) and (43) are guaranteed to hold by virtue of (11). 
Finally, singular 1-soliton solution, of second type, in birefringent fibers is given by

$$
q(x, t)=\sqrt{A_{1}\{1+\operatorname{coth}[B(x-v t)]\}} e^{i\left(-\kappa_{1}+\omega_{1} t+\sigma_{1}\right)}
$$

and

$$
r(x, t)=\sqrt{A_{2}\{1+\operatorname{coth}[B(x-v t)]\}} e^{i\left(-\kappa_{2}+\omega_{2} t+\sigma_{2}\right)}
$$

\section{CONCLUSION}

This paper filled in the gap on the study of solitons in birefringent fibers with perturbation terms and spatio-temporal dispersion. The singular soliton solutions, of second type, are obtained in this paper by the aid of ansatz method. The two types of nonlinear media that are studied in this paper are Kerr law and parabolic law. The results come with their corresponding constraints that must hold for the soliton solutions to exist.

These results are encouraging towards future goals. The solutions of this paper can serve as a foundation stone for analytical studies of optical rogons. An additional prospect of this work is to extend to DWDM systems. This is just a tip of the iceberg.

\section{ACKNOWLEDGMENT}

The fourth author (AB) and sixth author (MB) were supported by Qatar National Research Fund (QNRF) under the grant number NPRP 6-021-1-005, and the second author (QZ) was funded by the National Science Foundation of Hubei Province in China under the grant number 2015CFC891. The fourth author (AB) would like to thank Tshwane University of Technology during his academic visit in 2016. The fifth author (SPM) would like to thank the research support provided by the Departmentment of Mathematics and Statistics at Tshwane University of Technology and the support from the South African National Foundation under Grant Number 92052 IRF1202210126. 


\section{References}

[1] Bhrawy, A. H., Alshaery, A. A., Hilal, E. M., Savescu, M., Milovic, D. M., Khan, K. R., Mahmood, M. F., Jovanoski Z. and Biswas. A. "Optical solitons in birefringent fibers with spatio-temporal dispersion”, Optik, 125(17), pp. 4935-4944 (2014).

[2] Biswas, A. and Konar, S. "Introduction to non-Kerr law optical solitons", CRC Press Boca Raton, FL, USA (2006).

[3] Biswas, A., Khan, K., Rahman, A., Yildirim, A., Hayat, T. and Aldossary, O. M. "Bright and dark optical solitons in birefringent fibers with Hamiltonian perturbations and Kerr law nonlinearity", J Optoelectron Adv M., 14(7-8), pp. 571-576 (2012).

[4] Chen, Y. H., Wang, J. L. and Tian, W. X. "Soliton coupling in birefringent optical fiber with third order dispersion", Optik, 120(5), pp. 212- 215 (2009).

[5] Geng, X. and Lv, Y. "Darboux transformation for an integrable generalization of the nonlinear Schrödinger equation”, Nonlinear Dynam., 69(4), pp. 1621-1630 (2012).

[6] Jovanoski, Z. and Rowland, D. R. "Variational analysis of solitary waves in a homogeneous cubic-quintic nonlinear medium" J. Mod. Optic., 48(7), pp. 1179-1193 (2001).

[7] Kohl, R., Biswas, A., Milovic, D. and Zerrad, E. "Optical soliton perturbation in a nonKerr law media”, Opt. Laser Technol., 40(4), pp. 647-662 (2008).

[8] Kumar, S., Singh, K. and Gupta, R. K. "Coupled Higgs field equation and Hamiltonian amplitude equation: Lie classical approach and G'/G-expansion method", Pramana, 79(1), pp. 41-60 (2012).

[9] Li, H. and Wang, D. "Dynamics of dark-bright vector solitons in a birefringent fiber", Chinese Opt. Lett., 5(9), pp. 504-506 (2007).

[10] Mahmood, M. F. and Qadri, S. B. "Modeling propagation of chirped solitons in an elliptically low birefringent single-mode optical fiber", J. Nonlinear Opt. Phys., 8(4), pp. 469475 (1999). 
[11] Milovic, D. and Biswas, A. "Bright and dark solitons in optical fibers with parabolic law nonlinearity", Serbian J. Electric. Eng., 10(3), pp. 365-370 (2013).

[12] Nathan, P. M. C., Kalyanasundaram, N. and Ravikumar, D. "Soliton propagation in birefringent fibers", Fiber Integrated Opt., 27(2), pp. 99-111 (2008).

[13] Nathan, P. M. C., Kalyanasundaram, N. and Krishnam, R. M. “Analysis of optical soliton propagation in birefringent fibers", Int. J. Microwave Opt. Tech., 4(6), pp. 368-373 (2009).

[14] Rajan, M. S. M., Hakkim, J., Mahalingam, A. and Uthayakumar, A. "Dispersion management and cascade compression of femtosecond non-autonomous soliton in birefringent fiber", Eur. Phys. J. D., 67, pp. 115 (2013).

[15] Rand, D., Glesk, I., Bre's, C-S., Nolan, D. A., Chen, X., Koh, J., Fleischer, J. W., Steiglitz, K. and Prucnal, P. R. "Observation of temporal vector soliton propagation and collision in birefringent fiber", Phys. Rev. Lett., 98, pp. 053902 (2007).

[16] Savescu, M., Khan, K. R., Naruka, P., Jafari, H., Moraru, L. and Biswas, A. "Optical solitons in photonic nanowaveguides with an improved nonlinear Schrödinger's equation”, $J$. Comput. Theor. Nanoscience., 10(5), pp. 1182-1191 (2013).

[17] Savescu, M., Khan, K. R., Kohl, R. W., Moraru, L., Yildirim, A. and Biswas, A. “Optical soliton perturbation with improved nonlinear Schrödinger's equation in nanofibers", $J$. Nanoelectron. Optoelectron., 8(2), pp. 208-220 (2013).

[18] Shahi, A. K., Singh, V. and Ojha, S. P. "Dispersion characteristics of electromagnetic waves in circularly cored highly birefringent waveguide having elliptical cladding”, Prog. Electromag. Res., 75, pp. 51- 62 (2007).

[19] Triki, H. and Biswas, A. "Dark solitons for a generalized nonlinear Schrödinger's equation with parabolic and dual-power law nonlinearities", Math. Mod. Meth. Appl. S., 34(8), pp. 958-962 (2011). 
[20] Xu, Y., Jovanoski, Z., Bouasla, A., Triki, H., Moraru, L. and Biswas, A. "Optical solitons in multi-dimensions with spatio-temporal dispersion and non-Kerr law nonlinearity". J. Nonlinear Opt. Phys., 22(3), pp. 1350035 (2013).

[21] Zhong, W. P. "Soliton propagation near zero-dispersion wavelength in birefringence fiber”, Commun. Theor. Phys., 33(1), pp.157-160 (2000).

[22] Zhou, Q., Yao, D. and Chen, F. "Analytical study of optical solitons in media with Kerr and parabolic law nonlinearities", J. Mod. Opt, 60(19), pp. 1652-1657 (2013).

[23] Zhou, Q., Yao, D., Chen, F. and Li, W. "Optical solitons in gas-filled, hollow-core photonic crystal fibers with inter-modal dispersion and self-steepening", J. Mod. Opt. 60(10), pp. 854-859 (2013).

[24] Zhou, Q. “Analytical solutions and modulational instability analysis to the perturbed nonlinear Schrödinger equation", J. Mod. Opt. 61(6), pp. 500-503 (2014).

[25] Zhou, Q. "Analytic study on solitons in the nonlinear fibers with time-modulated parabolic law nonlinearity and Raman effect”, Optik, 125(13), pp. 3142-3144 (2014).

[26] Zhao, H., Zhao, X., Hao, H. "Breather-to-soliton conversions and nonlinear wave interactions in a coupled Hirota system", Appl. Math. Lett. 61, pp. 8-12, (2016).

[27] Guo, R., Hao, H., Zhang, L. "Dynamic behaviors of the breather solutions for the AB system in fluid mechanics”, Nonlinear Dyn. 74, pp. 701-709, (2013).

[28] Guo, R., Hao, H. "Breathers and localized solitons for the Hirota-Maxwell-Bloch system on constant backgrounds in erbium doped fibers", Annals of Physics, 344, pp. 10-16, (2014). [29] Zhou, Q., Zhu, Q., “Optical solitons in medium with parabolic law nonlinearity and higher order dispersion”, Waves in Random and Complex Media, 25 (1), pp. 52-59, (2015). [30] Zhou, Q., Zhu, Q., Bhrawy, A., Moraru, L., Biswas, A. “Optical solitons with spatiallydependent coefficients by Lie symmetry", Optoelectronics and Advanced Materials-Rapid Communications, 8(7-8), pp. 800-803, (2014). 
[31] Zhou, Q., Liu, L., Liu, Y., Yu, H., Yao, P., Wei, C., Zhang, H. "Exact optical solitons in metamaterials with cubic-quintic nonlinearity and third-order dispersion", Nonlinear Dyn. 80(3), pp. 1365-1371, (2015).

[32] Zhou, Q., Zhu, Q., Liu, Y., Yao, P., Bhrawy, A., Moraru, L., Biswas, A. "Bright-Dark combo optical solitons with non-local nonlinearity in parabolic law medium”, Optoelectronics and Advanced Materials-Rapid Communications 8(9-10), pp. 837-839, (2014). 